
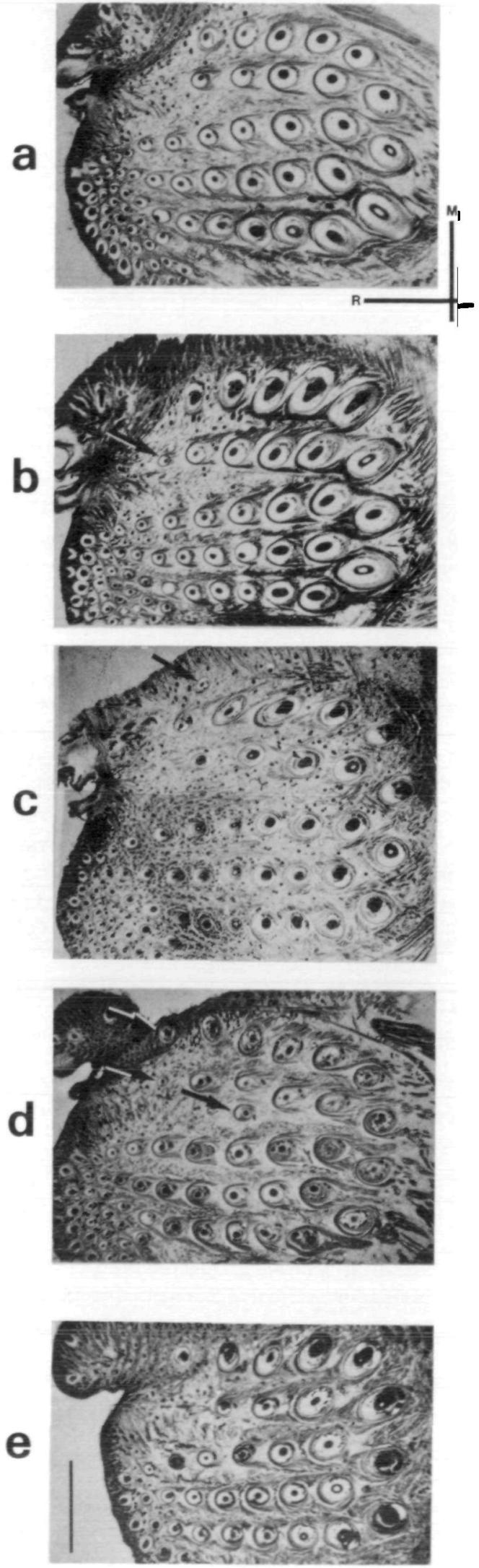
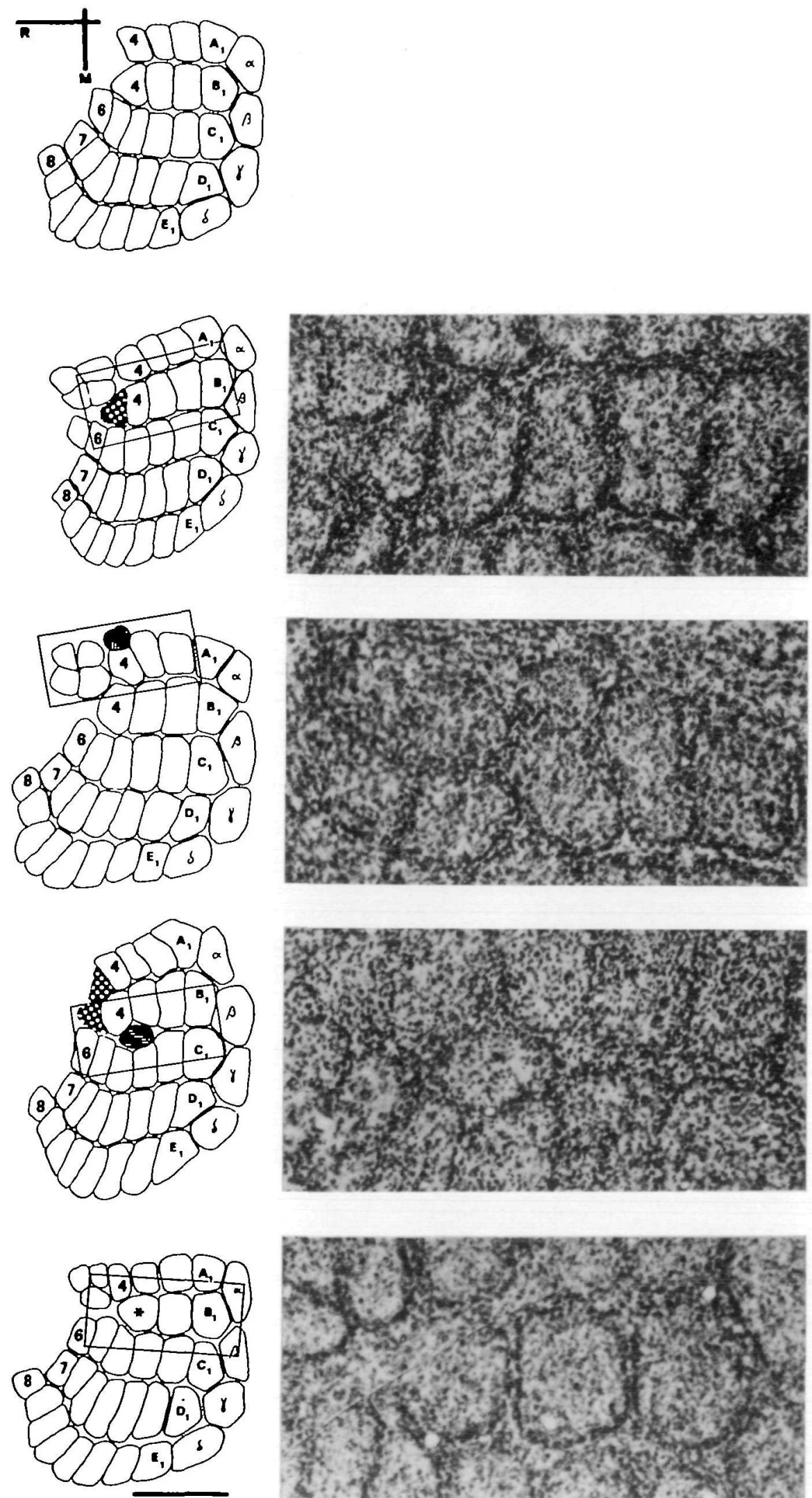

FIGURE 1 The left and middle columns show five sets of representations of whiskerpads and corresponding barrelfields. For the sake of comparison all whiskerpads are shown as if they are from the left side; all barrelfields, as if they are from the right side. Orientation is the same for all sets and is indicated by compasses in $a$ : $\mathrm{R}=$ rostral; $\mathrm{M}=$ mediodorsal; thus, for the barrelfield (camera lucida) drawings the brains are turned upside down: in neocortex, the somatosensory periphery is represented roughly upside-down. In all illustrations of left and middle column, rows $A$ are at the top, rows $\mathrm{E}$ at the bottom. $\alpha, \beta, \gamma, \delta$ are the caudal, "straddling" elements (see Figure $2 H$ and text). Numbers delimit standard field (see text) except for $e$ where the rostralmost element of B row 


\title{
Variation in pattern of mystacial vibrissae in mice
}

\author{
A quantitative study of ICR stock and several inbred strains
}

H. Van der Loos
J. Dörfl
E. Welker

The authors are affiliated with the Institute of Anatomy, University of Lausanne, Rue du Bugnon 9, 1011 Lausanne, Switzerland. This work was supported by the Swiss National Science Foundation (grants $3.776,3.516,3.238$ ), the Banque Cantonale Vaudoise, Emıl Barell-Stiftung ( $F$ Hoffmann-La Roche), Geigy Jubıläums-Stiftung, Sandoz-Stiftung zur Fōrderung der Medizinisch-Biologischen Wissenschaften, and Wild \& Leitz AG, Zürich. During part of this study E W. was the recipient of a NATO Science Fellowship from the Netherlands Organization for the Advancement of Purc Research (Z.W.O). The authors thank N. $V$ an der Loos for assistance throughout this study, M. C Cruz for histology, G. Escher for statustical, and E. J Gubbels (Central Institute for the Breeding of Laboratory Animals TNO, Zeist, The Netherlands) for genclic expertise, V.S. Caviness (Harvard University) and P. P. Giorg1 (University of Queensland) for critical discussion, $K$. Van der Saag for help with analysis and irtwork, K. Meler and $M$ Marfurt for help with animal kēeping, S. Daldoss for photography, and C. Vaclavik for typing. F. Andres provided Figure 6, and the management of Hôtel de la Forclaz, ambiance and shelter during the final analysis of the data and the preparation of the manuscript.

c) 1984, American Genetic Association

\begin{abstract}
We report on the variation in the pattern of mystacial vibrissae in ICR mice, of which one-half of the about 600 animals investigated showed one or more supernumerary whiskers (SWs). The SWs and their follicles--in all respects identical to the units of the standard pattern except for their smaller size-occurred at a restricted number of sites. In addition, a limited number of mice from two BALB/c strains were analyzed. Half of them also had one or more SWs, but mainly at one site. Mice of the C3H/HeJ and DBA/2J(a) strains were virtually without SWs, whereas animals from the NMRI strain were standard without exception. Nearly all animals of the C57BL/6J strain lacked between 1 and 4 vibrissae, always from one or two of the same adjacent sites. There was a slight overall predominance for the left side of the face to bear SWs and there was no clear-cut assoclation with sex; the entire population was without obvious defects. The sites where extra or lacking whiskers occur are associated with the lines of fusion between the medial and the lateral nasal fold, and between the latter and the maxillary arch. Where tested, we always found a topologic equivalency between the pattern of the whisker follicles and the contralateral pattern of the "barrels" - multineuronal units in layer IV of the parietal cerebral cortex - whether the pattern was standard, "enriched," or lacking in elements. The data presented in this paper provided a basls for several studies carried out subsequently on animals that are the offspring of those characterized here, studles suggesting that the occurrence of supernumerary and of lacking whiskers has a genetic basis.
\end{abstract}

THE PATTERN of the mystacial whiskers on the muzzle of the mouse is amazingly constant. "Amazing" when one considers vibrissae as banal hairs; not amazing, perhaps, when one considers vibrissae as structural entities that have "phylogenetic individuality" 3 much as, for example, the fingers of the hand. Our interest in that pattern was inspired by the existence of the barrelfield-the area of whisker representation in parietal neocortex ${ }^{26}$. In layer IV of that area, there are multineuronal units-barrels-arranged in a pattern that is homeomorphic with that of the whiskers on the whiskerpad. Whiskers are part of important tactile organs: each one of them issues from a sinus-hair follicle, a structure containing a high number of receptors. From each follicle, a follicular nerve arises that contributes to the formation of the infraorbital nerve, which, as part of the trigeminal nerve, enters the central nervous system (pons).

On the basis of differences in size and, particularly, shape of the barrels, a posteromedial barrel subfield (PMBSF) has been delineated: the cortical area where the large, posterior mystacial vibrissae project ${ }^{26}$. Further support for the distinction between the PMBSF and the anterolateral small-barrel- is marked by asterisk. In $b, c$, and $d$ supernumerary whiskers ( $S W s$ ) are indicated by arrows, supernumerary barrels (SBs) are hatched. Significant regions in drawings are boxed and represented as photomicrographs in right-hand column. In $a$ a normal pad and field are shown; in $b$, those that have a SW and corresponding SB at the B5 site; in $c$, those with a SW and a SB at the A4' site; in $d$, those with a SW and a SB at $\mathrm{C}^{\prime}$ and in positions $\mathrm{A} 5$, BS; in $e$, those with clement B4 missing. For all sets, muzzle and (contralateral) cortex stem from the same animal, except for $c$ where cortex is from a littermate of animal whose whiskcrpad is depicted. Whiskerpads are cut at $40 \mu \mathrm{m}$ and tangential to the surface of the skin, and stained with a modified Lillie technique. Barrelfields are cut at $40 \mu \mathrm{m}$, tangential to the surface of the cortex, and stained with methylene blue. Because of the curvature of the parietal cortex the barrelfields as depicted here have been taken from three or more serial sections. The bar in $e$ is $2 \mathrm{~mm}$ for the whiskerpad photographs in the lefthand column; the bar is $0.5 \mathrm{~mm}$ for the barrelfield drawings in the center column. 

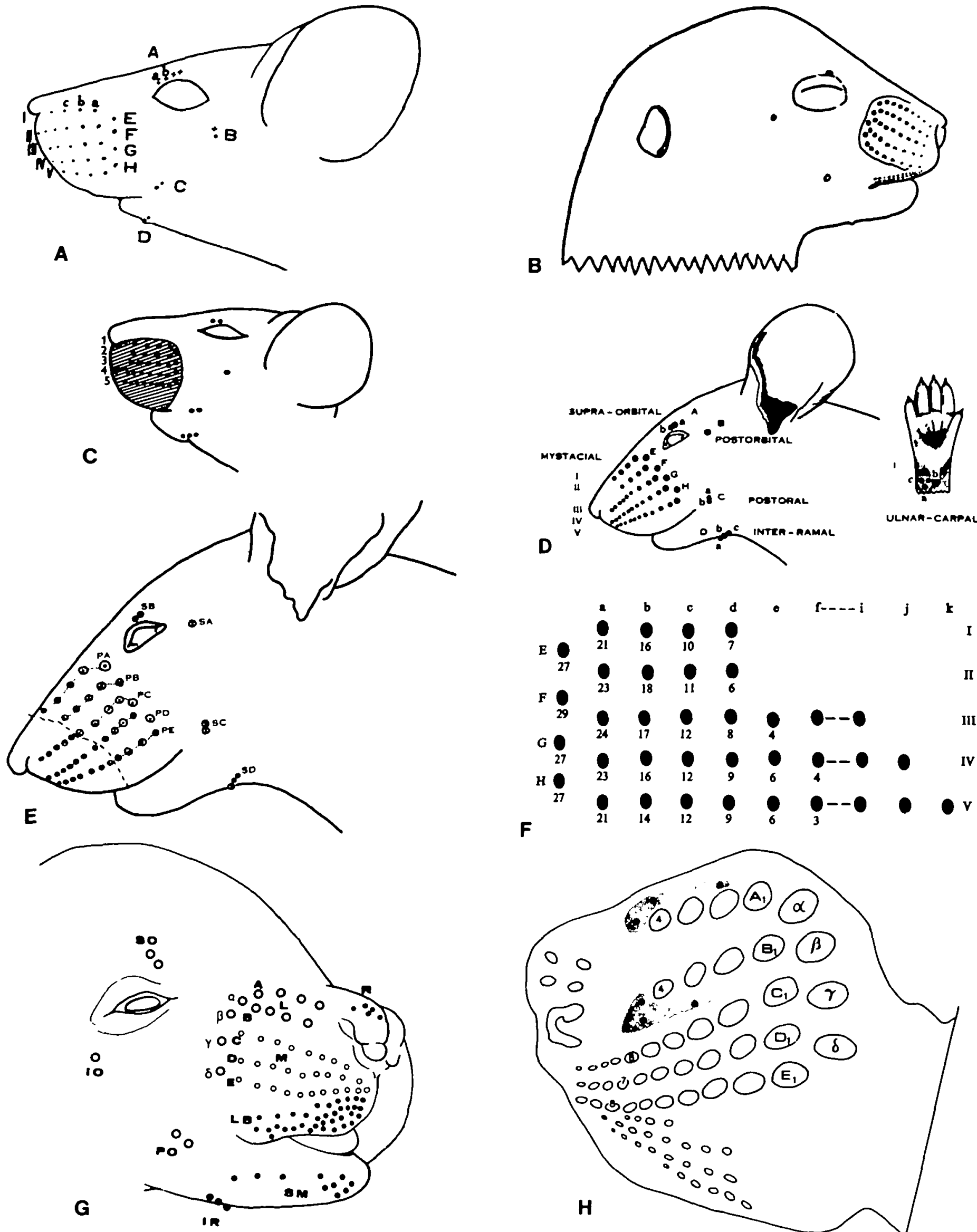
field came from physiological recordings (Nussbaumer and Van der Loos, ms. submitted). The barrels in the PMBSF receive only whisker input and no input from fur between the whiskers, whereas in the anterolateral small-barrelfield no such distinction could be made: it receives signals from both whiskers and intervening common fur.

For the purpose of the present paper we delimited a "standard" mouse whiskerpad. It bears 33 whiskers, 29 of which are aligned in five rows named A-E: 4 whiskers in the Arow, 4 in $B, 6$ in $C, 7$ in $D$, and 8 in the $E$ row, while four elements termed $\alpha, \beta, \gamma$, and $\delta$ are straddling the caudal ends of the rows. Figure $1 a$ shows the standard whiskerpad as well as the corresponding barrelfield. Figure $2 H$ shows the standard whiskerpad with the above nomenclature entered. These definitions of whiskerpad and barrelfield proved convenient in that large numbers of whiskerpads in newborn mice could be screened within a reasonable time while the corresponding cortical domain in the adult-roughly coinciding with the PMBSF-could be delineated consistently and with ease. This proved of particular importance for subsequent quantitative studies of the whiskerpad and the barrelfield, performed on several lines of mice that are the offspring of the animals discussed in the present paper 25 .

This article turns to the periphery only. Notwithstanding the constancy of the standard pattern, we as well as others have found variation in detail. The present paper is about that variation.

Several investigators have studied the arrangement of vibrissae of the mouse and, while trying to quantify it, were struck by its constancy. Often these studies considered not only the array of the so-called mystacial vibrissae (the "moustache") but also the other groups of them, all situated on the head and the neck

- FIGURE $2 A-G$ show reproductions of drawings accompanying the articles of authors who have described the pattern of vibrissal follicles in the mouse. $A$-Danforth ${ }^{3} ; B$-Grüneberg ${ }^{12} ; C$-Davidson and Hardy ${ }^{4} ; D$-Dun ${ }^{6} ; E$-Fraser and Kindred ${ }^{11}$; $F$-Ibrahim and Wright ${ }^{13}$; and $G-$ Yamakado and Yohro 29 . Sizes of drawings were adapted to fit the plate. $H$ shows our own scheme, derived from a section taken tangential to the skin of a left whiskerpad. It shows our nomenclature: lettered and numbered follicles delimit the pattern used as standard; shaded areas are the two zones of skin in which we found supernumerary whiskers. Black dots denote the sites in which we grouped the whiskers for the purpose of simplification (same convention as used in Figures 3 and 4): for the upper (medial) shaded zone these sites from caudal to rostral are $\mathrm{A} 2^{\prime}, \mathrm{A4}^{\prime}$, and $\mathrm{A} 5$; for the lower (lateral), $\mathrm{C3}^{\prime}, \mathrm{CS}^{\prime}$, and B5. These zones fall within the medial and the lateral "fusion lines" (see text). except for the ulnar-carpal vibrissae. Danforth's study ${ }^{3}$ of fur hairs and tactile vibrissae in the mouse pioneered in this direction. On the basis of a comparison of hair in mammals, feathers in birds, and scales in fish, he concluded that vibrissae - but not fur hairs-have what he called phylogenetic individuality.

In the following paragraphs we review the pertinent literature. Our own nomenclature is placed in brackets. Figure $2 A-G$ assembles the figures in which the listed authors summarize their findings; Figure $2 H$ illustrates our notation, based on an earlier study ${ }^{21}$.

Danforth $(1925)^{3}$, in the study referred to above, describes a vertical row of 4 whiskers termed E-H [our "straddlers" $\alpha-\delta$ ] and 5 horizontal rows named I-V. I [A] has 3,4 , or 5 hairs (4 is common) termed a,b,c, etc. (Figure $2 A$ ). Rows II-V [B-E] have a series of hairs that, going rostrad, gradually become smaller. Danforth chose to analyze 3 vibrissae per horizontal row; thus he counts until 19. He finds various exceptions to the pattern.

Grüneberg $(1943)^{12}$, in a study of anomalies associated with congenital hydrocephalus in mice, also states that in normal animals mystacial vibrissae occur in 5 rows. In hydrocephalic animals this pattern remained unchanged while other sets of whiskers did exhibit modifications. He does not make mention of the straddlers, but his Figure 1 clearly demonstrates their existence (Figure $2 B$ ). While the numbers of vibrissae per row are not given, the figure shows all five rows to contain 8 whiskers.

Davidson and Hardy $(1952)^{4}$ studied the development of vibrissae of the mouse in vivo and in vitro. They accept, with minor modifications as to the number of vibrissae per row, the pattern of the two preceding authors. Besides the vertical row of 4 large follicles [straddlers], they find in rows 1 [A] and 2 [B] always 4 follicles; in row $3[C]$, always 5 . In row 4 [D] they find at least 6 , and in row 5 [E] at least 8 follicles (Figure $2 C$ ).

$\operatorname{Dun}(1958)^{6}$, in his quantitative description of the various sets of vibrissae of the mouse, uses Danforth's terminology and characterizes the standard mystacial pattern as follows: rows I [A] and II [B] have 4 vibrissac each, while rows $I I I$ [C], IV [D], and V [E] average 10, 11 , and 11 vibrissae, respectively (Figure $2 D$ ). With Danforth, Dun recognizes a vertical row of 4 vibrissae [the straddlers]. This pattern and those of the other vibrissal groups (submental, post-oral, supra- and post-orbital, inter-ramal and ulnar-carpal) were to serve as baseline for breeding experiments in which the spatial distribution of the vibrissac was modified. In a subsequent study ${ }^{7}$ an attempt was made to define the time of action of two genes (tabby and raggy) that influence the development of vibrissae, and confirms essentially the standard pattern he had described before. The same can be said with regard to a next paper ${ }^{8}$ reporting on the results obtained through the introduction of these genes into the original stock.

Fraser and Kindred (1962) ${ }^{11}$, in a study that is a sequel to that of Dun and Fraser ${ }^{8}$, use a somewhat different system of notation, although their pattern is identical to that of Dun ${ }^{6}$. These authors, with regard to the mystacial vibrissae, draw into consideration vibrissae A 1-5 [ $\alpha$ and A 1-4], B 1-5 [ $\beta$ and B $1-4$ ], C 1-5 [ $\gamma$ and $C 1-4$ ], D $1-5$ [ $\delta$ and D 1-4], and E 1-4 [E 1-4] (Figure 2E). The basis for singling out these 4 or 5 posterior whiskers per row was that they are of about equal length and noticeably taller than those placed more anteriorly.

Ibrahim and Wright (1975) 13 , in an analysis of size and growth of the mystacial vibrissae in mice and rats, adopt Danforth's notation. They restrict their observations to the straddlers, to the 4 whiskers of our rows $A$ and $B$, to the 5 posterior elements of row $C$, and to the 6 posterior elements of rows $D$ and E (Figure $2 F$ ).

Yamakado and Yohro (1979) ${ }^{29}$, basing themselves on observations made in a number of mouse strains, confirm the vibrissal pattern as proposed by Dun ${ }^{6}$ and by Woolsey and Van der $\operatorname{Loos}^{26}$, while using the notation of the latter authors. On embryologic grounds, they divided the mystacial vibrissae into three groups: the lateral nasal, maxillary, and labial (the whiskerpad as defined in the present paper comprises elements of the first two groups). They described variations in number and arrangement of vibrissae and barrels. Rows $A$ and $B$, forming the lateral nasal group, each contain 4 whiskers, whereas rows $C, D$, and $E$, forming the maxillary group, bear 8,9 , and 11 vibrissae, respectively. In the view of these authors, straddlers $\alpha$ and $\beta$ probably belong to the lateral nasal group; $\gamma$ and $\delta$, to the maxillary group (Figure $2 G$ ).

It was a chance observation of an additional barrel in an odd place that, upon checking, turned out to be accompanied by an extra whisker in the corresponding place of the whiskerpad ${ }^{22}$, that set off the present investigation: a quantitative study of the variation in the pattern of mystacial vibrissae in mice from different populations. These populations were an outbred stock, ICR, and five inbred strains: albinos, BALB/c and NMRI; black, C57BL/6J; dilute brown, DBA/2J(a); and agouti, $\mathrm{C} 3 \mathrm{H} / \mathrm{HeJ}$.

Arguments, based on the occurrence of supernumerary whiskers (SWs) and corresponding barrels, led to the hypothesis that the peripheral pattern is responsible for the es- 
tablishment of the patterns in the central nervous system. The timing of critical, developmental events (peripheral pattern originates first, "brain maps" later) ${ }^{22}$ and the fact that the SWs occur only in the facial lines of fu$\operatorname{sion}^{23}$ were used in the elaboration of this idea, which also was supported by the observation that skin from presumptive, never-innervated whiskerpad cultured on chorioallantoic membrane will grow a follicle pattern akin to that found in vivo ${ }^{1,2}$.

From animals described in the present paper, a selection was made that served as origin of several lines of mice that were inbred (mostly by brother-sister matings) for particular whisker patterns including the standard one (work to be reported in subsequent publications).

\section{Materials and Methods}

Six populations of mice were used:

1. ICR. From June 1977 to March 1978 we obtained 45 pregnant mice from the Institut für Zuchthygiene of the University of Zürich (8057 Zürich, Switzerland). This institute had obtained its stock in 1964 from the Charles River Mouse Farms (Wilmington, MA 01887, USA) where the line, known as CDl(ICR)BR, was outbred. In Zürich, the animals had been bred without a rigorous system, although full sib mating was carefully avoided, until January 1976. From that date, the animals were outbred according to Robertson 9 . The 45 litters that we analyzed contained a total of 597 mice. Littersize varied from 5 to 20.

2. $B A L B / C$. In January 1980 three pregnant females were obtained from the Institut fūr Humangenetik of the University of Heidelberg ( 6900 Heidelberg, West Germany). This strain, BALB/ $c \pi(d)$, had been inbred for more than 100 generations. They yielded a total of 13 young. In the same period we received two pregnant females from the Swiss Institute for experimental Cancer Research ("ISREC," 1066 Epalinges, Switzerland). Their animals, from BALB/cByJ stock, are inbred. The stock had been obtained three generations earlier from the Jackson Laboratory. (Bar Harbor, Maine 04609, USA). The two females gave birth to a total of 11 young.

3. $\mathrm{C} 3 \mathrm{H} / \mathrm{HeJ}$. In March 1980 we obtained one couple from the Heidelberg Institute. Animals were from a strain that had been inbred for more than 100 generations. It generated 28 young.
4. C57BL/6J. From January to March 1980 , three pregnant females were obtained from the Heidelberg Institute. The strain had been inbred for more than 100 generations. They yielded a total of 30 young.

5. $D B A / 2 J(a)$. From January to March 1980 , eight pregnant females were obtained from Heidelberg. Strains had been inbred for more than 100 generations. There were 31 young from these females.

6. NMRI. In February 1980 one couple was received from Heidelberg. Animals were from a strain that had been inbred for five generations. The couple yielded a total of 13 young.

The evaluation procedure was identical for all animals. It followed usually within 24 hours after birth and was made, with the aid of a dissecting microscope, on all newborns with intact faces, living (almost all) or dead; their bodies, including the muzzles, at that time are still free of common fur. While we aimed at listing, for each animal, all deviations from the standard whisker pattern, our evaluation was directed to the following points (see Figure $2 H$ ):

1. In a standard whiskerpad, rows $A$ and $B$ have 4 vibrissae each. For these rows, with one exception, the supernumerary elements were situated at the rostral ends of the rows and termed A5 and B5. Whenever elements were missing, it was from loci A4 and B4.

2. Two other narrow strips of skin in which extra elements were observed, were 1) just medial to row $\mathrm{A}$-vibrissae here were called $A^{\prime}$; and 2 ) medial to row $C$ (i.e., between rows $C$ and $B$ )-these vibrissae were called $C^{\prime}$. The localization of $A^{\prime}$ and $C^{\prime}$ whiskers was further specified by recording the position of the extra element with reference to the whiskers in the row adjacent and lateral to it. In the strip of skin medial to row A the SWs occurred in positions between $\mathrm{A} 2^{\prime}$ and $\mathrm{A} 4^{\prime}$; in the strip medial to row $C$, between $C 2,3^{\prime}$ and $C 6,7^{\prime}$. We simplified notation by grouping SWs in $A^{\prime}$ loci into two sets: $\mathrm{A} 2^{\prime}$ and $\mathrm{A}^{\prime}$; and those in $\mathrm{C}^{\prime}$ loci into two sets: $\mathrm{C3}^{\prime}$ and $\mathrm{CS}^{\prime}$.

3. Three odd cases could not be classified according to 1 and 2 . These were of paired vibrissae, i.e., two equally large hairs issuing from one double follicle.

We did not count the total number of whiskers in rows $C, D$, and $E$ : they are numerous (about 10 per row) and the rostral ones are densely spaced and very small. This is the one restriction in our evaluation.

No SWs were seen between rows A and B,
C and D, and D and E, and between the straddlers.

The sex of 95 of the ICR animals as well as in all those of the inbred strains was determined by measuring the distance between anus and urogenital orifice.

Animals of different variations in whisker pattern were prepared for histology: they were anesthetized with Nembutal i.p. and perfused with 10 percent neutralized formalin in 0.9 percent $\mathrm{NaCl}$. Brain and whiskerpads were removed and postfixed in the same solution. The brain was bisected in the mid-sagittal plane, embedded in celloidin, and the hemispheres were cut tangentially to the pial surface over the barrelfield ${ }^{17}$; whiskerpads were soaked in sucrose and cut on a cryostat. The brains were Nissl-stained for cell bodies (methylene blue); whiskerpads were stained for myelin according to a modified Lillie method (Cruz et al., ms. in preparation) or with Mallory-azan.

\section{Results}

Over 700 newborn mice have been screened for their whisker patterns. A summary of our observations is given in Table $\mathrm{I}$.

The ICR animals showed the largest variety in deviations from the standard: all deviations and several combinations of them were observed, with the exception of the missing A4 and B4 that, when it occurred (it occurred in all litters), was the unique character of $\mathrm{C} 57 \mathrm{BL} / 6 \mathrm{~J}$. The only deviation in $\mathrm{C} 3 \mathrm{H} / \mathrm{HeJ}$ was an occasional A5 and in DBA/2J(a), an

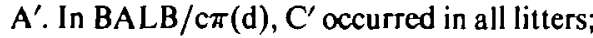
in $B A L B / c B y J$, in one. In each one of the BALB/c strains, one B5 occurred. NMRI mice were invariably standard.

When surveying all the mice screened we can add that in all but two litters with deviations there was one or more than one animal with the standard pattern and, although not the subject of specific investigation, no obvious defects were observed.

The data on the ICR animals-the most numerous lot-are presented in detail in Figure 3. Of the 45 litters analyzed, only one consisted of animals $(n=13)$ that showed no deviation from standard, while all others had one or more animals with one or more than one A5 and/or B5. For 28 of these, A5 and/or B5 whiskers were the only deviation from standard. Nine litters had one or more animals with one or more than one $\mathrm{C}^{\prime}$; two litters had one or more animals with one or more than one $\mathrm{A}^{\prime}$. Each one of the remaining five litters contained animals of which some had one or more $A^{\prime}$ 's and of which others had one or more C's, or, in one case, an animal with SWs in both positions, or animals that had a double follicle 
(from which issued two whiskers). Of the total of 37 animals with A's and/or C's, 20 had one or more than one A5 and/or B5 as well, and 17 had not.

With respect to consistency in the site of SWs expressed by a particular litter, those litters that showed SWs in the A5 position only are most numerous. Among those there are four with a (marked) tendency to have these whiskers to the left (Figure 3, nos. 05, 09, 12, and 21). There were no such litters with an opposite tendency. Although there are animals with SWs in the B5 site only, this was never the characteristic of an entire litter. Three litters showed a high overall occupancy of sites A5 and B5 (Figure 3, nos. 27, 28, and 29). Such a disposition was even more marked in litters that had one or more than one animal with $\mathrm{C}^{\prime}$ loci occupied (Figure 3, nos. 42, 35, 39, 45). This tendency also is illustrated by com- paring the mean number of A5 and/or B5 whiskers per animal belonging to a group of litters that have only A5 and/or B5 whiskers (Figure 3, nos. 02-29), with that belonging to the group of litters that have one or more than one $C^{\prime}$ (Figure 3, nos. 35-45). This value is for the first group 0.67 , and for the second group 1.21. Litters containing, besides whiskers A5 and B5, also and only those in $\mathrm{A}^{\prime}$ loci, do not show a higher incidence of $A 5$ and B5 whiskers than litters with A5 and B5 whiskers only.

Figure 4 summarizes all data; in it the animals are not grouped by litter, but first by stock and strains, and then by phenotype (whisker pattern).

When tested, the barrel pattern, deviation and all, was homeomorphic with respect to the contralateral whisker pattern. Some of these cases are illustrated in Figure $1 b-e$. It should be noted here that the SWs as well as the barrels associated with them, were identical to the elements belonging to the standard pattern, except for their size (they were smaller). Notably, the whisker follicles were provided with muscle slings (for description of this general feature see Dörfl ${ }^{5}$ ) and caused the SWs to whisk in harmony with surrounding vibrissae. SWs, or lacking whiskers at certain loci, often occurred on one side only, and although there were litters that for one location (A5) showed a left preference, no clear overall asymmetries were noted (Figures 3 and 4).

For the ICR stock, sex was determined for 15 percent of the young; for the other strains, all animals were sexed. The male/female ratio of the 220 sexed animals was 1.04:1. We did not observe a link between sex and the occurrence of SWs and of lacking whiskers, although in the animals with $\mathrm{C}^{\prime}$ whiskers females dominated (17 females, 7 males); in those with $\mathrm{A}^{\prime} \mathrm{s}$, males dominated (8 males, 2 females).

\section{Discussion}

This paper is on supernumerary whiskers, and on lacking ones, on the whiskerpad of the mouse. A characteristic of the distribution of these special tactile hairs over the surface of the whiskerpad, an elevated patch of skin, is its constant pattern. It is highly regular, and arguments have been advanced to state that the pattern is responsible for that of "barrels" in the brain $1,2,22,23$.

For the present study the muzzles of some 700 newborn mice were inspected. It was found that about half of the animals of ICR stock and of the BALB/c strains carry one or more supernumerary whiskers. In DBA/2J(a) we found one animal with a SW; in $\mathrm{C} 3 \mathrm{H} / \mathrm{HeJ}$, two, but the total number of animals was small. One strain (NMRI) showed only the standard pattern, while in another
(C57BL/6J) most animals lacked certain whiskers. In what follows we shall advance arguments for the notion that there is a genetic

Table II. Expected and observed occurrences in four sets of phenotypes of the ICR population involving the $\mathrm{A}^{5}, \mathrm{~B} 5, \mathrm{~A}^{\prime}$, and $\mathrm{C}^{\prime}$ vibrissae, respectively. The left hand column lists the phenotypes per set, i.e., no whisker of given type; presence of whisker on left; presence of whisker on right; presence of whiskers on both sides. The expected values were obtained by multiplying the number of individuals out of the total ICR population with the given

character on the left, with the number of those with the same character on the right, and dividing the product by the total number of animals

\begin{tabular}{|c|c|c|c|c|}
\hline & Phenotype & $\begin{array}{c}\text { Expected } \\
\text { values }\end{array}$ & $\begin{array}{c}\text { Observed } \\
\text { values }\end{array}$ & $\chi^{2}$ \\
\hline \multirow[t]{4}{*}{ A5 } & $-1-$ & 295.4 & 345 & $8.33^{* * *}$ \\
\hline & A5/- & 131.6 & 82 & $18.69^{* * *}$ \\
\hline & $-/ A 5$ & 117.6 & 68 & $20.92^{* * *}$ \\
\hline & A5/A5 & 52.4 & 102 & $\frac{46.95^{* * *}}{94.89 * * *}$ \\
\hline \multirow[t]{4}{*}{ B 5} & $-1-$ & 479.9 & 496 & 0.54 \\
\hline & BS/- & 71.1 & 55 & 3.65 \\
\hline & $-/$ B5 & 40.1 & 24 & $6.46^{*}$ \\
\hline & B5/BS & 5.9 & 22 & $\frac{43.93 * * *}{54.58 * * *}$ \\
\hline \multirow[t]{4}{*}{$\underline{\mathrm{A}^{\prime}}$} & $-1-$ & 584.1 & 586 & 0.01 \\
\hline & $\mathrm{A}^{\prime} /-$ & 6.9 & 5 & 0.52 \\
\hline & $-/ \mathbf{A}^{\prime}$ & 5.9 & 4 & 0.61 \\
\hline & $\mathrm{A}^{\prime} / \mathrm{A}^{\prime}$ & 0.1 & 2 & $\frac{36.10^{* * *}}{37.24 * * *}$ \\
\hline \multirow[t]{4}{*}{$\underline{C}^{\prime}$} & $-1-$ & 566.4 & 570 & 0.02 \\
\hline & $C^{\prime} /-$ & 11.6 & 8 & 1.12 \\
\hline & $-/ C^{\prime}$ & 18.6 & 15 & 0.70 \\
\hline & $C^{\prime} / C^{\prime}$ & 0.4 & 4 & $\frac{32.40 * * *}{34.24 * * *}$ \\
\hline
\end{tabular}

* Rejection at $P \leq 0.05$ of null hypothesis that the presence of a given vibrissa on one side is independent of the presence of the same element on the other $* * * P \leq 0.005$

Table I. Summary of numbers of various supernumerary whiskers (SWB) and lacking whiskers (-) in the mouse strains studied. Each strain is represented by a vertical bar. Data pertain to an analysis of 723 mice. The SWs are listed in the left-hand column (see Figure $2 H$ for locations of A5, B5, $\mathrm{A}^{\prime}, \mathrm{C}^{\prime},-\mathrm{A4}$ and -B4. $\mathrm{A}^{\prime}$ whiskers were grouped together, as were the $\mathrm{C}^{\prime} \mathrm{s}$. The "oddities" are $\mathrm{SW}$ s mentioned in text (see page 330 , pt. 3) and defined in

Figures 3 and 4. Numbers on the left and right sides of each vertical bar correspond to the total number of a particular SW on the left and right whiskerpad, respectively. $S_{1}$ gives totals of $S W_{s}$ per side; $S_{2}$, the sums of those totals; $n_{1}$ is the number of animals analyzed; $n_{2}$, the number of animals with SWs

\begin{tabular}{|c|c|c|c|c|c|c|c|c|c|c|c|c|}
\hline & \multicolumn{2}{|c|}{ ICR } & \multicolumn{2}{|c|}{ BALB/ck(d) } & \multicolumn{2}{|c|}{ BALB/cByJ } & $\mathrm{C} 3 \mathrm{H} / \mathrm{HCJ}$ & \multicolumn{2}{|c|}{$\mathrm{C} 57 \mathrm{BL} / 6 \mathrm{~J}$} & DBA $/ 2 \mathrm{~J}(\mathrm{a})$ & \multicolumn{2}{|c|}{ NMRI } \\
\hline A5 & $18 \overline{4}$ & {$[170$} & & & & & \begin{tabular}{l|l}
2 &
\end{tabular} & & & & & \\
\hline B5 & 77 & 46 & 1 & & & 1 & & & & & & \\
\hline $\mathbf{A}^{\prime}$ & 7 & 6 & & & & & & & & 1 & & \\
\hline $\mathrm{C}^{\prime}$ & 13 & 21 & 4 & 9 & & 2 & & & & & & \\
\hline Oddities & 3 & 0 & & & & & & & & & & \\
\hline$-A 4$ & & & & & & & & 10 & 15 & & & \\
\hline$-B 4$ & & & & & & & & 8 & 16 & & & \\
\hline$S_{1}$ & 284 & 243 & 5 & 9 & 0 & 3 & $2 \mid 0$ & 18 & 31 & \begin{tabular}{l|l}
0 & 1
\end{tabular} & 0 & 0 \\
\hline$S_{2}$ & & 27 & 1 & 4 & & 3 & 2 & 4 & 9 & $i$ & & 0 \\
\hline$n_{1}$ & & 97 & 1 & 3 & & 1 & 28 & & 0 & 31 & 1 & 3 \\
\hline$n_{2}$ & & 96 & 1 & 1 & & 2 & 2 & & 6 & 1 & & 0 \\
\hline
\end{tabular}




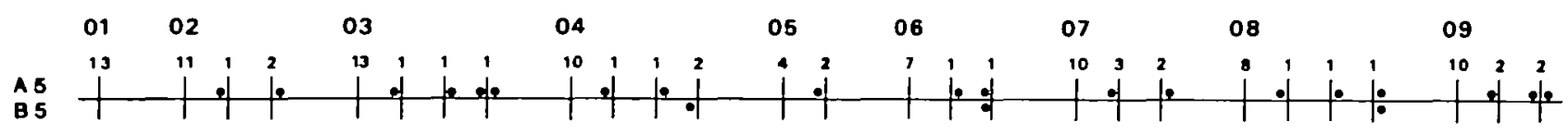

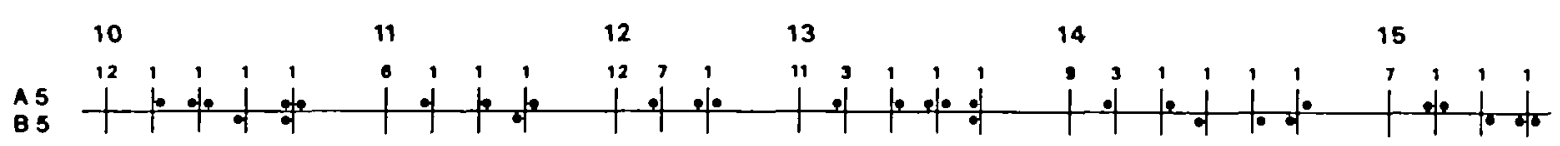

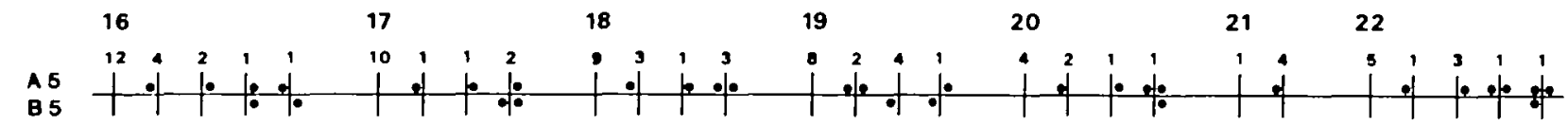

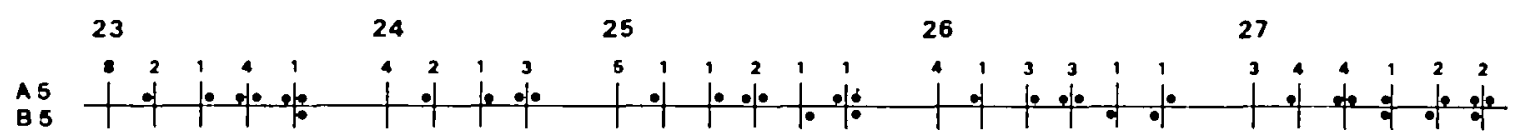

$28 \quad 29$

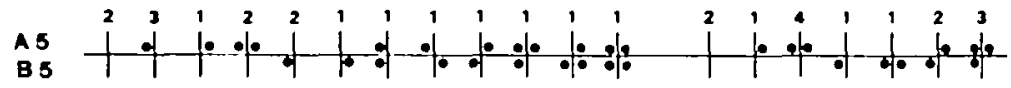
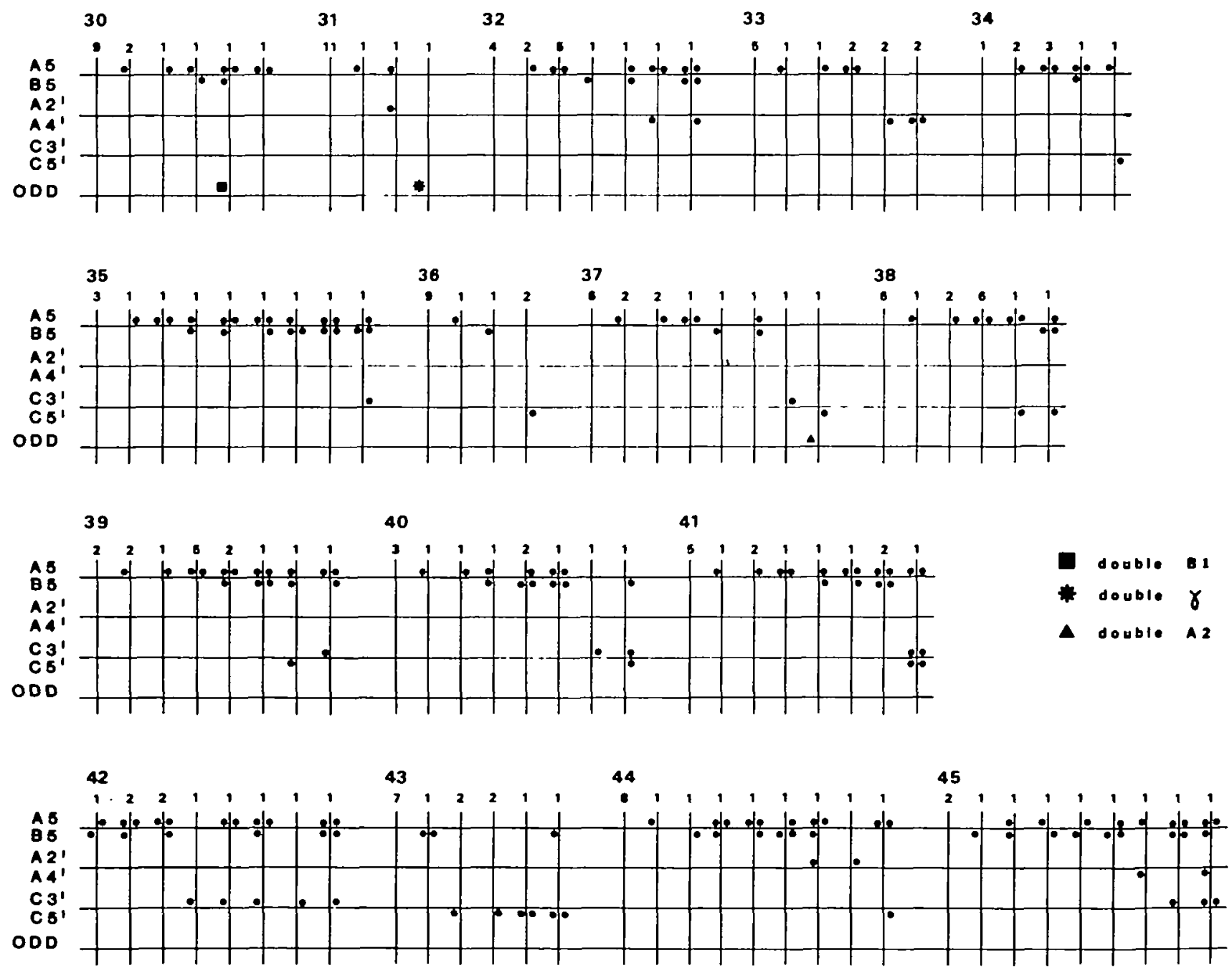

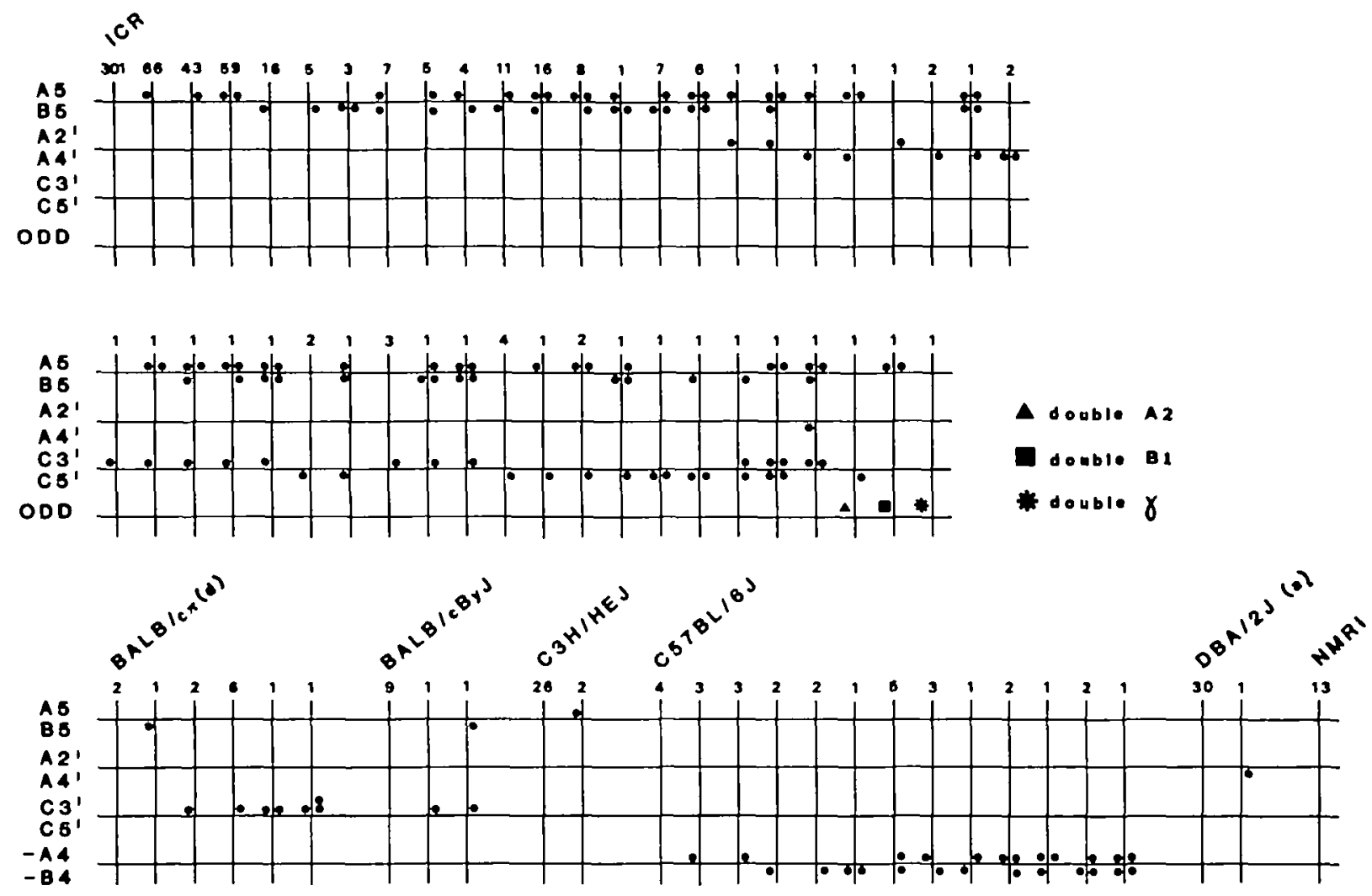

FIGURE 4 For the ICR line and for each of the strains studied, mice were grouped together according to the localization of their supernumerary whiskers (SWs), listed in the left hand column. ODD indicates double vibrissae (see page 330, pt. 3); key is to the right of the second staff. Each vertical bar represents a whisker pattern, characterized by the distribution of its SWs. As in Figure
3, a SW is represented by a dot; those left of a bar refer to left whiskerpads; those right of a bar, to right whiskerpads. The numbers on top of the vertical bars give the number of animals having a particular distribution of SWs over their two whiskerpads. For each group of mice, the first vertical bar - the bar without dotsrepresents the animals without $S W s$. basis for the occurrence of SWs and of lacking vibrissae.

Screening the data on the ICR stock for bilateral asymmetries in the distribution of SWs, we note a modest overall preference for SWs to occur on the lefthand side: the left1 right ratio $(\mathrm{LRr})$ is 1.17 . For two sites there

- FIGURE 3 Graphic representation of 45 litters of a nimals of the ICR stock. Litter numbers are in bold characters. Column on the left lists sites of supernumerary whiskers (SWs). For each litter, the individuals are grouped according to phenotype, i.e., - the distribution of SWs over their two whiskerpads; each group is represented by a vertical bar, the number at the top of which signifies the number of individuals within a particular group. A phenotype is expressed so that dots on the left side of a bar represent SWs on the left whiskerpad, and dots on the right side, on the right whiskerpad. The sites are given, at the corresponding horizontal level, in the left hand column. ODD refers to double vibrissae (see text p. 330, pt. 3; key is to the right of the eighth staff). Vertical bars without dots represent animals without SWs. The litters are arrayed according to the relative number of SWs per litter, first for litters that have $A 5$ and/or BS vibrissae only, then for those with $A^{\prime} s$, with $C^{\prime} s$, and finally with $A^{\prime} s$ and $C^{\prime} s$ and oddities. does appear to be preference: LRr for B5 is 1.67; for $C^{\prime}, 0.62$ (Table I). Although the numbers are small, it is interesting that in the BALB/c strains, $C^{\prime}$ whiskers also occur preferentially to the right $(\mathrm{LRr}=0.36)$. For ICR, the LRr for AS whiskers is 1.08, while it is 1.53 for animals in which A5 is the only site for SWs to occur. There are four litters with A5 vibrissae only that are asymmetric to the left. This latter observation seemed to point to a genetic influence, but see below. The question naturally arises if having a SW at a given location on one side is linked with an increased chance of having an identical one on the other side. Using A5-the most frequently occurring SW in the ICR population-as the example, we presumed that the occurrence of that whisker on one side is independent from its occurrence on the other. We then calculated the probability to have: 1) no AS on either side, 2) an A5 on the left, 3) an A5 on the right side, and 4) an A5 on both sides (see Table II). Comparing the observed with the expected values we note that for groups 2 and 3 the observed values are about one-half the expected ones, and that, for group 4 , the ob- served value is two times the expected one. Thus, it would appear that the occurrences of A5 whiskers on the left and on the right sides are not independent, i.e., the tendency to achieve a symmetric arrangement-none or two A5s-is greater than that of making an asymmetric arrangement. The same tests were performed on the other SWs: B5, $A^{\prime}$ and $C^{\prime}$. They led to similar results.

A5 and B5 are by far the most frequently occurring SWs among mice of the ICR stock. In a minority of animals with these vibrissae, there are one or more $A^{\prime} s$ and/or C's as well. However, since $A^{\prime}$ 's and $C^{\prime}$ 's may occur as sole SWs, it cannot be stated that the presence of $A^{\prime} s$ and/or C's in an animal is dependent on that of SWs in A5 and/or B5 sites. However, the higher incidence of A5 and B5 vibrissae in the litters in which one or more animals have one or more C's as compared to that of litters with A5 and/or B5 only, suggests a link between SWs AS and BS on one hand and C's on the other. For BALB/c, whose SWs are nearly always exclusively $C^{\prime}$ 's, such an association does not appear to exist.

The data on all animals whose sex had been 
determined, taken together, do not allow us to suggest a clear-cut association between $\alpha$ currence and site of SWs, and sex. Our material would seem to be too limited to draw conclusions from the dominance of females and males among animals with $\mathrm{C}^{\prime}$ and $\mathrm{A}^{\prime}$ whiskers, respectively.

The too-many and the too-few whiskers were associated with specific sites and thus we confirmed, on this ample material, observations made earlier $22,23,29$. The SWs are in locations A5 and B5, as well as in two narrow strips of skin, one at about the center of the whiskerpad, the other near its medial border. We have suggested 23,24 that these sites are associated with the lines of fusion (or of mergence $^{15,19}$ ) between the maxillary arch and the lateral nasal fold (B5 and the $C^{\prime}$ whiskers) and between the lateral and medial nasal folds (A5 and the $A^{\prime}$ whiskers). In an attempt to gain insight about the interaction between the presence of whisker B5 and that of C's at the same site (lateral line of fusion) and between the presence of B5 and that of $A^{\prime}$, we formulated the null hypothesis that the presence of one element of a pair is independent of that of the other. Expected values were obtained using contingency tables. Table III shows this analysis for pair B5-C' to the left, contrasted with pair B5- $\mathrm{A}^{\prime}$, also on the left. The null hypothesis was tested using the chi-square test and, while for the B5-C' pair the null hypothesis had to be rejected at $P=0.003$, suggesting a strong interaction between the presence of these elements, the test for the B5- $\mathrm{A}^{\prime}$ pair made independence highly likely $(P=0.21)$.

We then tested, in the same manner, all pairs of vibrissae, both ipsi- and contralateral with respect to each other. Figure 5 summarizes the departures from independence stating the probabilities by which the null hypotheses had to be rejected. Whereas, on the left side the occurrence of elements in the same lines of fusion show strong interaction, this was less marked for B5-C' and not present at all for $A 5-A^{\prime}$. It appears that the presence of A5 and B5 on both sides as well as across the midline, is strongly interactive for all four locations.

It is of interest to note that the vibrissae that may be lacking (in the $\mathrm{C} 57 \mathrm{BL} / 6 \mathrm{~J}$ strain) are A4 and B4, which are close to the medial and lateral fusion lines. For a description of facial morphogenesis in the mouse, see Tamarin and Boyde 20 .

Comparing observed and expected values for all 28 contingency tables (of which only those for the B5-C' and the B5- $\mathrm{A}^{\prime}$ on the left side are shown in Table III) we noted that for phenotypes with only one $S W$ of the combination in question the observed values were nearly always lower than the expected ones; this held for all pairs having A5 and/or B5 whiskers, while for phenotypes with none or two elements present the observed values were higher. We conclude that, for any animal, the presence of one $S W$ favors that of another one.

In the literature there are depictions of several barrelfields in which supernumerary barrels (SBs) can be seen. These are at the most common site, A5. Since a SB is always accompanied by a $\mathrm{SW}$ (as is a $\mathrm{SW}$ by a $\mathrm{SB}$ ), we may conclude that here we were confronted, too, with the existence of SWs (e.g., possibly Woolsey and Van der Loos ${ }^{26}$, Fig. 14, 9L; Rice and Van der Loos ${ }^{18}$, Fig. 1; Jeanmonod et al. ${ }^{14}$, Table I). Incidental illustrations of whiskerpads with an A5 are in Woolsey et al. ${ }^{27}$, Fig. 5, bottom left, and in Jeanmonod et al. ${ }^{14}$, Fig. 3 .

Also, there are reports that explicitly state the occurrence of SWs. In an analysis of the variation in the whisker pattern occurring in 165 mice of undefined stock, Danforth ${ }^{3}$ reports 1) a single occurrence of $A^{\prime}$ whiskers, 2 on each side; 2) an occasional absence of an $\mathrm{A4}$, and a $\mathrm{SW}$ in the A-row; 3) a single oc-

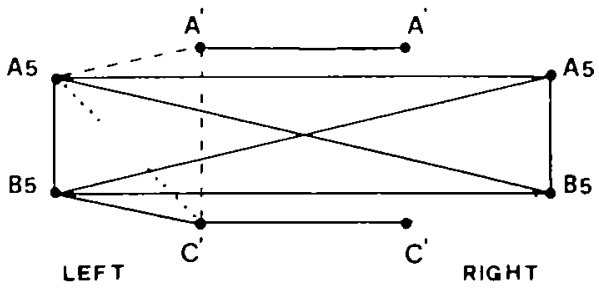

FIGURE 5 Summary of 28 tests of independence based on contingency tables (cf. Table III) and performed for each of the pairs that can be formed between whiskers $\mathrm{A} 5, \mathrm{~B} 5, \mathrm{~A}^{\prime}$ and $\mathrm{C}^{\prime}$ on the left and right side. Whiskers are represented by heavy dots. The continuous lines indicate departure from independence at $P \leq 0.005$; the broken lines, at $P$ $\leq 0.025$; the stippled lines, at $P \leq 0.050$. Where no lines exist, whiskers of the pair in question occur independently.

currence of a double $\alpha$ whisker; and 4) one occurrence of a missing D3 whisker. Dun ${ }^{6}$ in an analysis of 306 mice, presumably the $F_{1}$ of an inbred CBA line and an albino stock, found 3 whiskerpads with a missing A4 and/or B4 whisker. Ibrahim and Wright ${ }^{13}$ report that in less than 1 percent of an unspecified number of mice of the $\mathrm{C} 3 \mathrm{H}$ strain studied, "one vibrissal follicle [was] missing." These authors, in a parallel study of "closed colony Wistarderived albino rats," found a double $\gamma$ whisker in 25 percent of an unspecified number of animals. In some litters, all young had this anomaly and this suggested a genetic basis to these authors.

Yamakado and Yohro ${ }^{29}$ confirmed the occurrence of SWs in the $C^{\prime}$ site as described by $u^{22}$ (they did not find them in the $A^{\prime}$ row). They suggested, independently from $V$ an der $\operatorname{Loos}^{23}$, that SWs are formed at the lines of fusion as discussed above. It is of special interest that their animals with $\mathrm{C}^{\prime}$ whiskers were of the BALB/c strain, mice which in our material, too, had C's as almost their only SWs.

Table III. Contingency table on the left relates to whisker pair B5-C'; on the right to pair B5-A'. Both pairs are from the left muxzle; on the right muzxle any or no supernumerary whisker may have occurred. The key is in the center square: $a$ indicates the phenotype and shows the presence or absence of the whisker in question on the left muzzle; $b$ shows the expected occurrence of the phenotype in question, i.e., the product of the numbers given in the corresponding squares of top row and left column, divided by the total number, 597, of ICR animals analyzed; $c$ shows the observed occurrence of the same phenotype

\begin{tabular}{|c|c|c|c|c|c|c|c|c|c|c|c|}
\hline & & no $C^{\prime}$ & 585 & $\mathrm{C}^{\prime}$ & 12 & & & no $A^{\prime}$ & 590 & $\mathbf{A}^{\prime}$ & 7 \\
\hline no B5 & 520 & $\begin{array}{l}\text { no } B 5 \\
\text { no } C^{\prime}\end{array}$ & $\begin{array}{r}509.55 \\
513 \\
\end{array}$ & $\begin{array}{c}\text { no } B 5 \\
C^{\prime}\end{array}$ & $\begin{array}{c}75.45 \\
72 \\
\end{array}$ & a & b & $\begin{array}{l}\text { no } B 5 \\
\text { no } A^{\prime}\end{array}$ & $\begin{array}{c}513.90 \\
515 \\
\end{array}$ & $\begin{array}{c}\text { no } B 5 \\
A^{\prime}\end{array}$ & $\begin{array}{r}76.10 \\
75 \\
\end{array}$ \\
\hline$\underline{\text { B5 }}$ & 77 & $\begin{array}{c}\text { B5 } \\
\text { no } C^{\prime}\end{array}$ & $\begin{array}{r}10.45 \\
7\end{array}$ & $\begin{array}{l}\text { BS } \\
C^{\prime}\end{array}$ & $\begin{array}{c}1.55 \\
5\end{array}$ & & & $\begin{array}{c}B 5 \\
\text { no } A^{\prime}\end{array}$ & $\begin{array}{r}6.10 \\
5\end{array}$ & $\begin{array}{l}\text { B5 } \\
A^{\prime}\end{array}$ & $\begin{array}{r}0.90 \\
2\end{array}$ \\
\hline
\end{tabular}



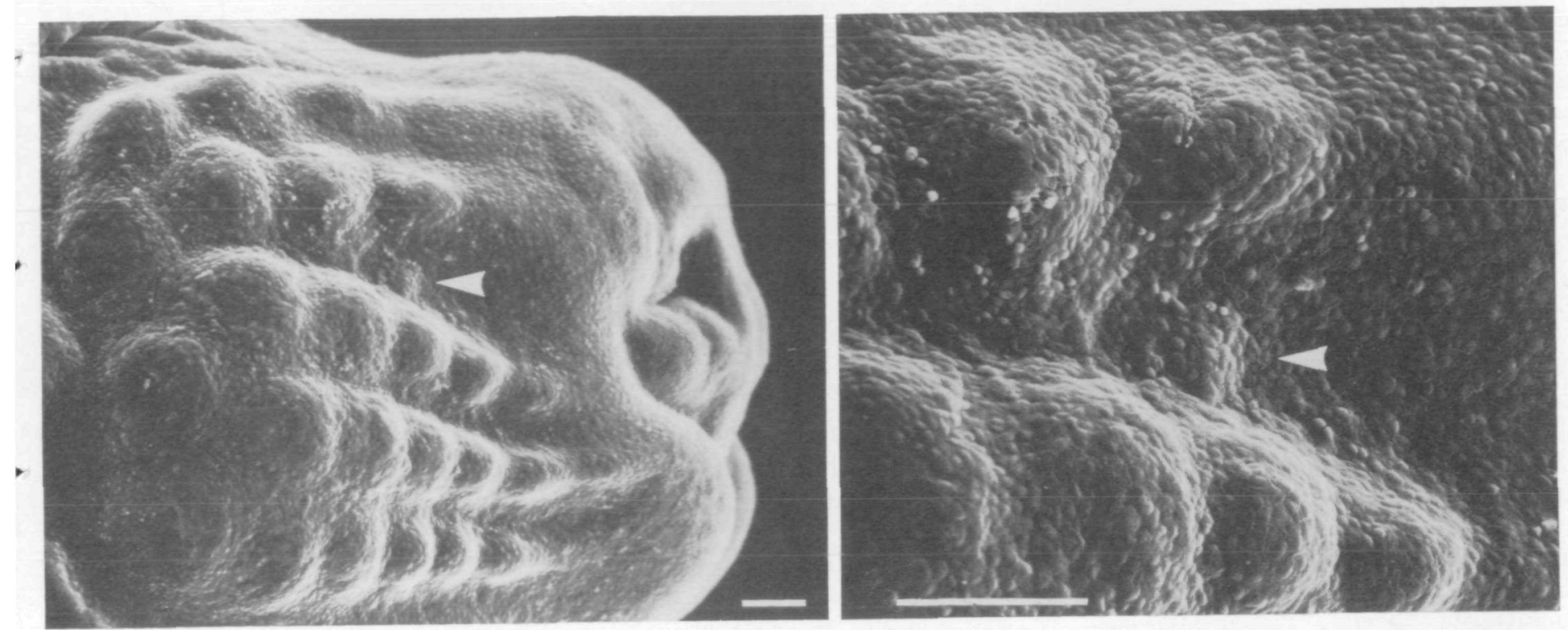

- FIGURE 6 Scanning electron mictographs from right muzzle taken from a mouse fetus of 13 days gestational age (conception is on day 0). Rostral is to the right; dorsal, up. Right micrograph is detail from center of pad, showing the rudiment of a supernumerary whisker at site C3' (arrowheads), situated between row $\mathrm{C}$-the middle row of follicle rudiments-and row B. Fetus was obtained through hys- terectomy performed under deep Nembutal (i.p.) anesthesia of the pregnant fermale, fixed in glutaraldehyde 2 percent in $0.1 \mathrm{M}$ phosphate buffer at $\mathrm{pH} 7.2$, critical point dried and sputter-coated with gold. Bars: $0.1 \mathrm{~mm}$. (Micrographs courtesy of Dr. F. Andrés.)
Our one animal outside the ICR strain (which had SWs in all listed sites) that had an $\mathrm{A}^{\prime}$ was from the DBA/2J(a) strain, while an A5 was seen in a $\mathrm{C} 3 \mathrm{H} / \mathrm{HeJ}$ mouse. NMRI had no SWs at all. We agree with Yamakado and Yohro $^{29}$ that the preferential occurrence of certain SWs in certain populations of mice speaks for a genetic component in determining whether or not a mouse may bear SWs. But we do not agree with them when they deduce that SWs are determined epigenetically on the grounds that they only occur at fusion lines (the "intermediate zones between growth centers" 29 ) and can occur unilaterally (see last paragraph of Discussion). They studied 5 $d d / y$, a BALB/c $(n u /+), 7$ BALB/c $(n u / n u)$, and 5 hairless $(h r / h r)$ mice, of which the first and the last are of unknown origin. In the first set there were no SWs; in the second, 3 ; in the third, 3; and in the fourth, 5. Their ratio $\mathrm{SWs} /$ mice screened, for BALB/c, is comparable to ours for $B A L B / c B y J$, but not for $B A L B / c_{\pi}(d)$, which had a higher ratio.

Yamakado and Yohro ${ }^{29}$ also used ICR mice, but those were part of an analysis of the development of vibrissae by scanning electron microscopy (SEM). Their ICR mice seem not to have borne SWs for if they had, some of the SEM specimens-particularly those of gestational day 13 and older-would have shown them and, if they had, the authors certainly would have seen them. Figure 6 is a scanning electron micrograph from a descendent of the ICR group described in the present paper, showing the primordium of a $\mathrm{C}^{\prime}$ whisker.
There seems to be much variation between subgroups belonging to the same strain, or to the same stock of mice. But the variation appears to be in the relative number of animals with SWs and not in the site of the SWs with respect to BALB/c: their and our strains had their $C^{\prime}$ s in the 3,4 place! SWs must have been rare in Japan; the authors report having examined more than 100 individuals of an additional 11 strains with negative results.

While it may not be surprising that highly inbred strains have $S W$ s at a particular location, or lack whiskers at particular locations, it is unexpected that within one strain some animals have SWs and some do not; obviously, there is interindividual variability. Possibly, a SW may be considered a threshold character ${ }^{28}$ with expression based on residual genetical heterogeneity ${ }^{10}$. In addition, environmental factors may play a role; in fact they must be of prime importance if the population is homogeneous. The limited degree of variation in sites of SWs within the different inbred strains, and the wide variation in the outbred ICR stock, speak in favor of a genetic basis for these variations and, thereby, for the existence of SWs. With respect to the variation within the ICR stock, it is striking that there appears to be a clustering of given phenotypes in certain litters: there are litters with a high number of $C^{\prime}$ whiskers; there are those with a high number of A5 and B5 whiskers; and there are even litters that show a strong left-side preference for the occupancy of the A5 site.

We have tried to estimate the probability of "clustering" occurring by chance by comparing a hypothetical population in which certain phenotypes were randomly distributed with the observed one. We took as an example all the $34 \mathrm{C}^{\prime}$ whiskers, divided over 27 animals. There are 597 animals in our population and 45 litters (mean size 13.22). Our null hypothesis was that these SWs are randomly distributed over the 45 litters whose mean size was rounded off to 13 ; such a distribution is binomial. In Table IV we give the expected and observed number of litters with $0,1,2$, and

Table IV. Goodness-of-fit test (chi-square) of expected and observed numbers of litters in which $0,1,2$, and $3 \mathrm{C}^{\prime}$ whiskers occurred in an average litter of 13 animals. The real population consisted of 597 mice bearing a total of $34 \mathrm{C}^{\prime}$ whiskers distributed over 45 litters. The expected numbers follow a binomial distribution with an estimated probability of 34/597. Eight-digit numbers were used for computation

\begin{tabular}{cccc}
\hline $\begin{array}{c}\text { No. } C^{\prime} \\
\text { whiskers } \\
\text { per litter }\end{array}$ & $\begin{array}{c}\text { Exp. } \\
\text { freq. }\end{array}$ & $\begin{array}{c}\text { Obs. } \\
\text { freq. }\end{array}$ & \multicolumn{1}{c}{$\chi^{2}$} \\
\hline 0 & 21.00 & 33 & $6.86^{* *}$ \\
1 & 16.48 & 3 & $11.03^{* * *}$ \\
2 & 5.97 & 4 & 0.65 \\
3 & 1.55 & 5 & $7.72^{* *}$ \\
& 45.00 & 45 & $26.26^{* * *}$ \\
\hline
\end{tabular}

* Rejection at $P \leq 0.01$ of null hypothesis that $C^{\prime}$ whiskers follow a binomial distribution ***P$\leq 0.005$ 
3 or more $C^{\prime}$ vibrissae. The goodness-of-fit test (chi-square) showed that the observed distribution would occur by chance only with a probability lower than 0.005 . Hence, we must reject our null hypothesis. We conclude that the clustering of $C^{\prime}$ vibrissac within particular litters is not due to random distribution.

When we consider the unit of analysis not to be the whisker itself, but an animal with one or more $C^{\prime}$ whiskers, then, following a similar analysis, we find that such "enriched" mice are clustered within particular litters, now with a confidence level of $P \leq 0.01$.

A final analysis concerned the asymmetrical distribution of A5 whiskers. As stated, there were four litters with a high left-right ratio but taking as units of analysis animals with an A5 whisker to the left as the sole character we had to accept the null hypothesis, recognizing the observed distribution of the animals in question to be a random event.

The existence of clustering - be it of given supernumerary whiskers or of animals of given phenotype-does not form a decisive argument in favor of genetic or epigenetic factors determining the occurrence of supernumerary whiskers.

In conclusion, we propose that there is a systematic basis for the occurrence of supernumerary whiskers, and we presume that factors involved in the formation of them act on the fusion of the nasal folds and the maxillary arch. Beside the localization of their action, the analysis of the data for the ICR population leads us to distinguish three groups of factors. 1) A general factor that determines the presence of mystacial SWs regardless of side. It forms the basis for the observation that the incidence of one $S W$ is associated with an increased chance for a second one to occur. A similar, general factor determining the overall number of vibrissae belonging to a particular group of "secondary" vibrissae has been postulated ${ }^{16} .2$ ) Factors that specifically act on the different lines of fusion, again regardless of side. They underlie the interaction between the occurrence of several SWs (Figure 5), and also the clustering of $\mathrm{C}^{\prime}$ whiskers in some litters (Table IV). 3) A third factor deals with laterality. See the left-right differences in the occurrence of SWs at a particular site (Table I) and the mode of interaction between the occurrence of two SWs for several whisker combinations that is not the same for the left and the right side (Figure 5).

We suggest a genetic basis for these factors. Starting from the population of mice described in this paper, we succeeded in selectively breeding animals (over an average of 15 generations) for particular whisker patterns, nonstandard and standard. The results of these ongoing experiments will be the subject of subsequent articles. For a preliminary report see Van der Loos et al. ${ }^{24}$.

\section{References}

1. ANDRES, F. L. and H. VAN DER LOOS. Whisker patterns form in cultured non-innervated muzzle skin from mouse embryos. Neurosci. Lett. 30: $37-41.1982$.

2. Cultured embryonic non-innervated mouse muzzle is capable of generating a whisker pattern. Int. J. Devl. Neurascience 1: 319-338. 1983.

3. DANFORTH, C. H. Hair in its relation to questions of homology and phylogeny. Am. J. Amat. 36: 47-68. 1925.

4. DavidSON, P. and M. H. HARDY. The development of mouse vibrissae in vivo and in oitro. J. Anat. 86:342-356. 1952.

5. DORFL, J. The musculature of the mystacial vibrissae of the white mouse. J. Anat. 135:147-154. 1982.

6. DUN, R. B. Growth of the mouse coat. VI. Distribution and number of vibrissae in the house mouse. Austr. J. Biol. Sci. 11:95-105. 1958.

7. - The development and growth of vibrissac in the house mouse with particular references to the time of action of the tabby ( $\mathrm{Ta}$ ) and ragged ( $\mathrm{Ra}$ ) genes. Austr. J. Biol. Sci. 12:312-330. 1959.

8. - and A.S. FraSER. Selection for an invariant character, vibrissa number, in the house mouse. Austr. J. Biol. Sci. 12:506-523. 1959.

9. FALCONER, D. S. Genetic aspects of breeding methods. In UFAW Handbook. A. N. Norden and W. Lane-Petter, Eds. 3rd ed. Livingstone, Edinburgh and London. p. 72-96. 1967.

10. Festing, M. F. W. Inbred Strains in Biomedical Research. MacMillan, London and Basingstoke. 1979.

11. Fraser, A. S. and B. M. KINDRed. Selection for an invariant character, vibrissa number, in the house mouse. III. Correlated responses. Austr. J. Biol. Sci. 15:188-206. 1962.

12. GRUNEBERG, H. Congenital hydrocephalus in the mouse, a case of spurious pleiotropism. J. Genet. 45:1-21. 1943.

13. IBRAHIM, L. and E. A. WRIGHT. The growth of rats and mice vibrissae under normal and some abnormal conditions. J. Embryol. Exp. Morph. 23:831-844. 1975 .

14. JEANMONOD, D., F. L. RICE, and H. VAN DER LoOS. Mouse somatosensory cortex: alterations in the barrelfield following receptor injury at different early postnatal ages. Neuroscience 6: 1503-1535. 1981.
15. Patten, B. M. The normal development of the facial region. In Congenital Anomalies of the Face and Associated Structures. S. Pruzansky, Ed. Thomas, Springfield, Illinois. p. 11-45. 1961.

16. PenNycuik, P. R. and J. M. Rendel. Selection for constancy of score and pattern of secondary vibrissae in $T a / T a-T a / Y$ and $T A /+$ mice. Austr. J. Biol. Sci. 30:303-317. 1977.

17. RICE, F. L. and D. ANDERS. A small guillotine to prepare brains for sectioning in defined, add planes. Neurosci. Lelt. 6:157-163. 1977.

18. —_ and H. VAN DER LOOS. Development of the barrels and barrel field in the somatosensory cortex of the mouse. J. Comp. Neurol. 171:545-560. 1977.

19. Streeter, G. L. Description of age groups XV, XVI, XVII, and XVIII, being the third issue of a survey of the Carnegie Collection. Contributions to Embryology 32:133-203, Carnegie Institution of Washington Publication 575. 1948. In Developmental Horizons in Human Embryos; Age Groups XI to XXIII, Embryology Reprint Volume II, G. L. Streeter, Ed. Carnegie Institution of Washington, Washington, D.C. 1951.

20. TAMARIN, A. and A. BoYDE. Facial and visceral arch development in the mouse embryo: a study by scanning electron microscopy. J. Anat. 124: 563-580. 1977.

21. VAN DER LOOS, H. and T. A. WOOLSEY. SOmatosensory cortex: structural alterations following early injury to sense organs. Science 179:395-398. 1973.

22. — and J. DORFL. Does the skin tell the somatosensory cortex how to construct a map of the periphery? Neurosci. Lett. 7:23-30. 1978.

23. - The development of topological equivalencies in the brain. In Neural Growth and Differentiation. E. Meisami and M. A. B. Brazier, Eds. Raven Press, NY. p. 331-336. 1979.

24. - J, J. DÓRFL, and E. WELKER. Selective breoding for variation in number of mystacial vibrissae in mice; consequences for the cortical barrelfield. Neurosci. Leit. suppl. 14:S384. 1983.

25. WELKER, E. and H. VAN DER LOOS. Genetically determined supernumerary mystacial vibrissae in albino mice (ICR): association with peripheral innervation and with size of barrelfield. Neurascl. Lett. suppl. 14:S401. 1983.

26. WOOLSEY, T. A. and H. VAN DER LOOS. The structural organization of layer IV in the somatosensory region (SI) of mouse cerebral cortex: the description of a cortical field composed of discrete cytoarchitectonic units. Brain Res. 17:205-242. 1970.

27. - C. Welker, and R. H. Schwartz. Comparative anatomical studies of the $\mathrm{Sm} l$ face cortex with special reference to the occurrence of "barrels" in layer IV. J. Comp. Neurol, 164:79-94. 1975.

28. WRIGHT, S. An analysis of variability in number of digits in an inbred strain of guinea pigs. Gentics 19:506-551. 1934

29. YAMAKADO, M. and T. YoHRO. Subdivision of mouse vibrissae on an embryological basis, with descriptions of variations in the number and arrangement of sinus hairs and cortical barrels in BALB/c (nu/t; nude; $n u / n u)$ and hairless ( $h r / h r)$ strains. Am. J. Anat. 155:153-174. 1979. 